\title{
THE COMBINATORIAL STRUCTURE OF EVENTUALLY NONNEGATIVE MATRICES*
}

\author{
SARAH CARNOCHAN NAQVI ${ }^{\dagger}$ AND JUDITH J. MCDONALD ${ }^{\ddagger}$
}

\begin{abstract}
In this paper it is shown that an eventually nonnegative matrix $A$ whose index of zero is less than or equal to one, exhibits many of the same combinatorial properties as a nonnegative matrix. In particular, there is a positive integer $g$ such that $A^{g}$ is nonnegative, $A$ and $A^{g}$ have the same irreducible classes, and the transitive closure of the reduced graph of $A$ is the same as the transitive closure of the reduced graph of $A^{g}$. In this instance, many of the combinatorial properties of nonnegative matrices carry over to this subclass of the eventually nonnegative matrices.
\end{abstract}

AMS subject classifications. 15A18, 15A48, 15A21

1. Introduction. The Perron-Frobenius Theorem for irreducible nonnegative matrices has spawned a wealth of interesting ideas in the study of nonnegative matrices. Graph-theoretic spectral theory of matrices continues to develop, and in this paper we are interested in extending many of these ideas to the class of eventually nonnegative matrices. The relationship between the combinatorial structure of a nonnegative matrix and its spectrum, eigenvectors, and Jordan structure is surprisingly elegant and beautiful, as well as useful. Surveys of results of this type can be found in Berman and Plemmons [1], Hershkowitz [5], and Schneider [13].

Friedland [3], Handelman [4], Zaslavsky and Tam [16], and Zaslavsky and McDonald [15] have looked at extending some of these combinatorial ideas to eventually positive matrices and eventually nonnegative matrices. In their study they found examples of eventually nonnegative matrices for which the relationship between the combinatorial structure of the matrix, and its spectrum, eigenvectors, and Jordan structure was inconsistent with that of nonnegative matrices. For example, there are irreducible eventually nonnegative matrices for which the spectral radius is a multiple eigenvalue. Even when the spectral radius is a simple eigenvalue of an irreducible eventually nonnegative matrix, the associated eigenvector need not be positive. Moving to properties of reducible eventually nonnegative matrices, we see that the combinatorial spectral properties exhibited by reducible nonnegative matrices need not carry over to eventually nonnegative matrices.

In this paper we show that it is really the contributions from the nilpotent part of an eventually nonnegative matrix that determine whether or not the combinatorial structure will be an accurate predictor of its spectral properties. In Section 3, we show that if $A$ is an eventually nonnegative matrix that is nonsingular, or if all the Jordan blocks in the Jordan form of $A$ associated with the eigenvalue zero are $1 \times 1$, then there is a positive integer $g$ such that the transitive closure of the reduced graph

\footnotetext{
* Received by the editors on 9 July 2001. Final manuscript accepted for publication on 1 September 2002. Handling Editor: Shmuel Friedland.

$\dagger$ Department of Mathematics and Statistics, University of Regina, Regina, Saskatchewan, Canada, S4S 0A2 (carnoc@math.uregina.ca). Supported by an NSERC PGS-A Scholarship.

¥ Department of Mathematics, Washington State University, Pullman, WA 99164-3113, USA (jmcdonald@math.wsu.edu). Supported by an NSERC Operating Grant.
} 
of $A$ is the same as the transitive closure of the reduced graph of $A^{g}$.

In Section 4, we show that many of the combinatorial properties of reducible nonnegative matrices carry over to reducible eventually nonnegative matrices provided the index of zero is less than or equal to one.

Section 2 contains a list of the definitions and notation used in this paper.

2. Definitions and Notation. For the set $\{1, \ldots, n\}$, we write $\langle n\rangle$. The matrix $A \in \mathrm{M}_{n}(\mathbb{R})$ (the $n \times n$ matrices with real entries) is called:

(strictly) positive $(A \gg 0)$ if $a_{l j}>0$ for all $l, j \in\langle n\rangle$;

semipositive $(A>0)$ if $a_{l j} \geq 0$ for all $l, j \in\langle n\rangle$ and $A \neq 0$; and nonnegative $(A \geq 0)$ if $a_{l j} \geq 0$ for all $l, j \in\langle n\rangle$.

We say the matrix $A$ is eventually nonnegative if there exists a positive integer $N$ such that for all integers $g \geq N, A^{g} \geq 0$. The matrix $A$ is eventually positive if, in addition, $A^{g} \gg 0$ for all integers $g \geq N$.

Let $K, L \subseteq\langle n\rangle$. The matrix $A_{K L}$ is the submatrix of $A$ whose rows are indexed by $K$ and whose columns are indexed by $L$. The sets $K_{1}, K_{2}, \ldots, K_{k}$ partition a set $K$ if they are pairwise disjoint and $\bigcup_{j=1}^{k} K_{j}=K$. We allow $K_{j}=\emptyset$ for ease of notation in some places. If $\kappa=\left(K_{1}, \ldots, K_{k}\right)$ is an ordered partition of a subset of $\langle n\rangle$, we write

$$
A_{\kappa}=\left[\begin{array}{llll}
A_{K_{1} K_{1}} & A_{K_{1} K_{2}} & \ldots & A_{K_{1} K_{k}} \\
A_{K_{2} K_{1}} & A_{K_{2} K_{2}} & \ldots & A_{K_{2} K_{k}} \\
\vdots & \vdots & & \vdots \\
A_{K_{k} K_{1}} & A_{K_{k} K_{2}} & \ldots & A_{K_{k} K_{k}}
\end{array}\right]
$$

We say $A_{\kappa}$ is block lower triangular if $A_{K_{l} K_{j}}=0$ whenever $l<j$.

Let $A$ be a square, complex matrix. The spectrum of $A, \sigma(A)$, is the multiset consisting of the eigenvalues of the matrix. The spectral radius of $A, \rho(A)$, is the $\max _{\lambda \in \sigma(A)}|\lambda|$.

Consider the multiset $\sigma=\left\{\lambda_{1}, \ldots, \lambda_{n}\right\}$. Let $\bar{\sigma}$ be the multiset $\left\{\bar{\lambda}_{1}, \ldots, \bar{\lambda}_{n}\right\}$, where $\bar{\lambda}$ is the complex conjugate of $\lambda$. If $\bar{\sigma}=\sigma$, then we say the multiset is self-conjugate. Let $\rho(\sigma)=\max _{\lambda \in \sigma}|\lambda|$.

We let $\operatorname{mult}_{\lambda}(A)$ denote the degree of $\lambda$ as a root of the characteristic polynomial of $A$, and $\operatorname{index}_{\lambda}(A)$ denote the degree of $\lambda$ as a root of the minimal polynomial of $A$. Let $t=\operatorname{index}_{0}(A)$, and for each $i \in\langle t\rangle$, set $\eta_{i}(A)=\operatorname{nullity}\left(A^{i}\right)-\operatorname{nullity}\left(A^{i-1}\right)$. The sequence $\eta(A)=\left(\eta_{1}(A), \eta_{2}(A), \ldots, \eta_{t}(A)\right)$ is referred to as the height or Weyr characteristic of $A$.

Let $\eta=\left(\eta_{1}, \eta_{2}, \ldots, \eta_{t}\right)$ and $\nu=\left(\nu_{1}, \nu_{2}, \ldots, \nu_{t}\right)$ be two sequences of nonnegative integers. (Append zeros if necessary to the end of the shorter sequence so they are the same length.) We say $\nu$ is majorized by $\eta$ if $\sum_{l=1}^{j} \nu_{l} \leq \sum_{l=1}^{j} \eta_{l}$ for all $1 \leq j \leq t$ and $\sum_{l=1}^{t} \nu_{l}=\sum_{l=1}^{t} \eta_{l}$. We write $\nu \preceq \eta$.

The generalized eigenspace of the matrix $A$ corresponding to $\lambda$, denoted $E_{\lambda}(A)$, is the nullspace of $(A-\lambda I)^{r}$, where $r=\operatorname{index}_{\lambda}(A)$.

A Jordan chain corresponding to $E_{\lambda}(A)$ is a set of nonzero vectors $\{x,(\lambda I-$ A) $\left.x, \ldots,(\lambda I-A)^{r-1} x\right\}$, where $(\lambda I-A)^{r} x=0$. A Jordan basis for the generalized eigenspace of $A$ is a basis of $E_{\lambda}(A)$ consisting of the union of Jordan chains. 
We denote the $l \times l$ lower triangular Jordan block associated with the eigenvalue $\lambda$ by $J_{l}(\lambda)$ and write $J(A)$ to represent the Jordan canonical form of the matrix $A$. In order to make the distinction between a collection of Jordan blocks and a matrix in Jordan form, we denote the collection of Jordan blocks associated with the matrix $A$ by $\mathcal{J}(A)$. Notice that for $\lambda \neq 0, J_{l}(\lambda) \in \mathcal{J}(A)$ if and only if $J_{l}\left(\lambda^{g}\right) \in \mathcal{J}\left(A^{g}\right)$ and appears the same number of times. If the index $\operatorname{dex}_{0}(A) \geq 2$, then the Jordan structure corresponding to the eigenvalue 0 will be different between the two matrices.

Let $J$ be a matrix in Jordan canonical form. The collection of Jordan blocks $\mathcal{J}(J)$ is self-conjugate if whenever $J_{l}(\lambda) \in \mathcal{J}(J)$, the matrix $J_{l}(\bar{\lambda}) \in \mathcal{J}(J)$ and appears the same number of times.

All graphs in this paper are directed graphs. Let $\Gamma=(V, E)$ be a graph where $V$ is a finite vertex set and $E \subseteq V \times V$ is an edge set. A path from $j$ to $m$ is a sequence of vertices $j=v_{1}, v_{2}, \ldots, v_{t}=m$ with $\left(v_{l}, v_{l+1}\right) \in E$ for $l=1, \ldots, t-1$. A simple path is a path where the vertices are pairwise distinct. The empty path will be considered to be a simple path linking every vertex to itself. The path $v_{1}, v_{2}, \ldots, v_{t}$ is a cycle if $v_{1}=v_{t}$ and $v_{1}, v_{2}, \ldots, v_{t-1}$ is a simple path.

Let $\Gamma=(V, E)$ be a graph. We say a vertex $l$ has access to a vertex $j$ if there is a path from $l$ to $j$ in $\Gamma$. A vertex is final if it does not access any other vertex in $\Gamma$. A vertex is initial if it is not accessed by another vertex. We define the transitive closure of $\Gamma$ by $\bar{\Gamma}=(V, \bar{E})$ where $\bar{E}=\{(j, l) \mid j$ has access to $l$ in $\Gamma\}$. If $l$ has access to $j$ and $j$ has access to $l$, we say $j$ and $l$ communicate. The communication relation is an equivalence relation. Thus we can partition $V$ into equivalence classes which we will refer to as the classes of $\Gamma$.

We define the graph of a matrix $A$ by $G(A)=(V, E)$ where $V=\langle n\rangle$ and $(l, j) \in E$ whenever $a_{l j} \neq 0$.

A graph $\Gamma$ is said to be cyclically h-partite if there exists a partition of $V$ into $h$ nonempty sets, $V_{1}, . ., V_{h}$, such that each edge of $\Gamma$ is from $V_{i}$ to $V_{i+1}$ for some $i=1, \ldots, h$ where $V_{h+1}$ is taken to be $V_{1}$.

A square matrix $A$ is called $h$-cyclic if $G(A)$ is cyclically $h$-partite. In other words, there is an ordered partition of $\langle n\rangle$ into $\omega=\left(V_{1}, V_{2}, \ldots, V_{n}\right)$ such that

$$
A_{\omega}=\left[\begin{array}{ccccc}
0 & A_{V_{1} V_{2}} & 0 & \ldots & 0 \\
0 & 0 & A_{V_{2} V_{3}} & \ldots & 0 \\
\vdots & \vdots & \vdots & \ddots & \vdots \\
0 & 0 & 0 & \ldots & A_{V_{h-1} V_{h}} \\
A_{V_{h} V_{1}} & 0 & 0 & \ldots & 0
\end{array}\right] .
$$

The largest possible $h$ for which $A$ is $h$-cyclic is called the cyclic index of $A$.

Let $J$ be a matrix in Jordan canonical form with $\rho(J)>0$. The collection $\mathcal{J}(J)$ is $h$-cyclic if $J_{l}(\lambda) \in \mathcal{J}(J)$ implies $J_{l}\left(e^{\frac{2 \pi i}{h}} \lambda\right) \in \mathcal{J}(J)$ and appears the same number of times. For nonnegative matrices, this is equivalent to $J=J(A)$, where $A$ is $h$-cyclic. The largest $h$ for which $\mathcal{J}(J)$ is $h$-cyclic is called the cyclic index of $\mathcal{J}(J)$.

Let $\sigma=\left\{\lambda_{1}, \ldots, \lambda_{n}\right\}$ be a multiset of complex numbers. We say $\sigma$ is a Frobenius multiset if there exists $h \leq n$ such that

(i) $\rho(\sigma)>0$. 
(ii) $\sigma \cap\{z \in \mathbb{C}:|z|=\rho(\sigma)\}=\left\{\rho(\sigma), e^{\frac{2 \pi i}{h}} \rho(\sigma), e^{\frac{4 \pi i}{h}} \rho(\sigma), \ldots, e^{\frac{2(h-1) \pi i}{h}} \rho(\sigma)\right\}$.

(iii) $\sigma=e^{\frac{2 \pi i}{h}} \sigma$.

Let $\sigma$ be a Frobenius multiset. Let $h$ be the number of eigenvalues of maximum modulus $\rho(\sigma)$. Then $h$ is called the index of cyclicity of $\sigma$. We say $\sigma$ is a Frobenius multiset with cyclic index $h$.

Let $J$ be a matrix in Jordan canonical form. We say $\mathcal{J}(J)$ is a Frobenius collection if there exists a positive $h$ such that

(i) $\rho(J)>0$, there is exactly one Jordan block corresponding to $\rho(J)$, and this block is $1 \times 1$.

(ii) If $\lambda \in \sigma(J)$ and $|\lambda|=\rho(J)$, then $\lambda=\rho(J) \cdot\left(h^{\text {th }}\right.$ root of unity).

(iii) $\mathcal{J}(J)$ is $h$-cyclic.

We will refer to $\mathcal{J}(J)$ as a Frobenius collection with cyclic index $h$.

A square matrix $A$ is reducible if there exists a permutation matrix $P$ such that

$$
P^{T} A P=\left[\begin{array}{ll}
B & 0 \\
C & D
\end{array}\right],
$$

where $B$ and $D$ are nonempty square matrices. The matrix $A$ is irreducible if it is not reducible. Irreducibility is equivalent to the property that every two vertices in $G(A)$ communicate. The classes of $G(A)$ are also referred to as the irreducible classes of $A$.

Given a matrix $A$, there exists an ordered partition $\kappa=\left(K_{1}, K_{2}, \ldots, K_{k}\right)$ of $\langle n\rangle$ such that each $K_{i}$ corresponds to a class of $G(A)$ and $A_{\kappa}$ is block lower triangular. The matrix $A_{\kappa}$ is referred to as the Frobenius normal form of $A$. A class $K_{j}$ is said to be singular if $A_{K_{j} K_{j}}$ is singular and nonsingular otherwise.

We define the reduced graph of $A$ by $R(A)=(V, E)$ where $V=\{K \mid K$ is an irreducible class of $A\}$, and $E=\{(K, L) \mid$ there is edge from a vertex $j \in K$ to a vertex $l \in L$ in $G(A)\}$.

The singular length of a simple path in $R(A)$ is the sum of the indexes of zero of each of the singular vertices it contains. The level of a vertex $K$ is the maximum singular length over all the simple paths in $R(A)$ that terminate at $K$.

Let $\nu_{i}(A)$ be the number of singular vertices with level $i$ in $R(A)$ and let $m$ be the largest number for which $\nu_{i}(A) \neq 0$. Then $\nu(A)=\left(\nu_{1}(A), \ldots, \nu_{m}(A)\right)$ is referred to as the level characteristic of $A$.

3. The Relationship of the Frobenius Normal Form of an Eventually Nonnegative Matrix to the Frobenius Normal Form of its Powers. The nonnegative matrices exhibit many combinatorial properties that do not appear to carry over to the eventually nonnegative matrices. In this section we begin by showing how to construct an irreducible eventually nonnegative matrix for which the spectral radius is a multiple eigenvalue. We then show the nilpotent part of an eventually nonnegative matrix is the major contributor to the apparent lack of combinatorial consistency between nonnegative and eventually nonnegative matrices.

EXAMPLE 3.1. We take a reducible nonnegative matrix and turn it into an irreducible eventually nonnegative matrix by adding an an appropriate nilpotent matrix. 
Let

$$
B=\left[\begin{array}{llll}
1 & 1 & 0 & 0 \\
1 & 1 & 0 & 0 \\
1 & 1 & 1 & 1 \\
1 & 1 & 1 & 1
\end{array}\right]
$$

Then $B$ is a reducible nonnegative matrix with $\rho(B)=2$, a double root. Notice $x=[1,-1,0,0]^{T}$ is a right nullvector of $A$ and $y^{T}=[0,0,1,-1]$ is a left nullvector $A$. Let

$$
C=\left[\begin{array}{cccc}
0 & 0 & 1 & -1 \\
0 & 0 & -1 & 1 \\
0 & 0 & 0 & 0 \\
0 & 0 & 0 & 0
\end{array}\right]
$$

Then $B C=C B=C^{2}=0$. Let $A=B+C$. Then $A^{j}=B^{j}$ for all $j \geq 2$, so $A$ is eventually nonnegative. On the other hand,

$$
A=\left[\begin{array}{cccc}
1 & 1 & 1 & -1 \\
1 & 1 & -1 & 1 \\
1 & 1 & 1 & 1 \\
1 & 1 & 1 & 1
\end{array}\right]
$$

is irreducible with $\rho(A)=2$ appearing as an eigenvalue with multiplicity two. Notice $\operatorname{index}_{0}(A)=2$.

Now consider

$$
D=\left[\begin{array}{llll}
1 & 1 & 0 & 0 \\
1 & 1 & 0 & 0 \\
1 & 1 & 2 & 2 \\
1 & 1 & 2 & 2
\end{array}\right]
$$

Then $D+C$ is an irreducible eventually nonnegative matrix with the spectral radius 4 as a simple root. The associated eigenvector is $z=[0,0,1,1]^{T}$, which is not a positive vector.

Using this technique, we can create reducible eventually nonnegative matrices for which various combinatorial properties of nonnegative matrices fail, and we encourage interested readers to experiment on their own. We also present a few more examples at the end of our paper

We now investigate eventually nonnegative matrices for which the index of zero is at most one.

First, we choose a $g$ such that if a submultiset of $\sigma(A)$ raised to the $g^{\text {th }}$ power is a self-conjugate Frobenius multiset, then the submultiset itself is a self-conjugate Frobenius multiset.

Let

$$
\begin{gathered}
D_{A}=\left\{d \mid \theta-\alpha=\frac{c}{d}, \quad \text { where } r e^{2 \pi i \theta} \in \sigma(A), r e^{2 \pi i \alpha} \in \sigma(A), r>0\right. \\
\left.c \in \mathbb{Z}^{+}, d \in \mathbb{Z} \backslash\{0\}, \operatorname{gcd}(c, d)=1\right\} .
\end{gathered}
$$


$D_{A}$ compares the rational portion of the exponent for nonzero eigenvalues of the same modulus. Any nonrational differences are ignored. If no two eigenvalues of a matrix $A$ have the same modulus, or if the only eigenvalues of the same modulus are equal, then $D_{A}=\emptyset$.

By choosing a prime power $g$ that is not an element of $D_{A}$, the $g^{t h}$ powers of distinct elements in $\sigma(A)$ are distinct elements in $\sigma\left(A^{g}\right)$. For example, suppose $\sigma(A)=\{1,-1\}=\left\{e^{0}, e^{\frac{(2 \pi i)}{2}}\right\}$, then $D_{A}=\{ \pm 2\}$. In this instance, $\sigma(A)$ is a selfconjugate Frobenius multiset. The even powers of the eigenvalues of $A$ form two Frobenius multisets each with a value of 1 . The odd powers of the eigenvalues of $A$ are distinct and form a self-conjugate Frobenius multiset with the same cyclicity as $\sigma(A)$. In particular, if $g$ is any odd prime, $\lambda \in \sigma(A)$ and $\gamma \in \sigma(A)$, then $\lambda^{g}=\gamma^{g}$ if and only if $\lambda=\gamma$.

Lemma 3.2. Let $A \in M_{n}(\mathbb{R})$. Let $g$ be a prime number such that $g \notin D_{A}$. If $\lambda \in \sigma(A)$ and $\gamma \in \sigma(A)$ are such that $\lambda^{g}=\gamma^{g}$, then $\lambda=\gamma$.

Proof. Let $\lambda=r e^{2 \pi i \theta} \in \sigma(A)$. Let $\gamma$ be an element of $\sigma(A)$ such that $\gamma^{g}=\lambda^{g}=$ $r^{g} e^{2 \pi i g \theta}$. Then we can write $\gamma=r e^{2 \pi i \theta+\frac{2 \pi i k}{g}}=r e^{2 \pi i\left(\theta+\frac{k}{g}\right)}$ where $k \in\{0,1, \ldots, g-1\}$. If $k=0$, then $\gamma=\lambda$. Suppose $k \neq 0$. Consider the difference $\theta-\left(\theta+\frac{k}{g}\right)=-\frac{k}{g}$, which is in simplified form since $g$ is prime. Thus $g \in D_{A}$, a contradiction. Therefore $\gamma=\lambda$. $\mathrm{Q}$

Lemma 3.3 takes advantage of the irreducibility of the lower block in the $g^{\text {th }}$ power to show that the block above it is zero.

LEMMA 3.3. Let $A$ be an eventually nonnegative matrix such that index $x_{0}(A) \leq 1$. Let $g$ be a prime number such that $g \notin D_{A}$ and $A^{s} \geq 0$, for all $s \geq g$. If $A^{g}$ is reducible, let $\omega=\left(W_{1}, W_{2}\right)$ be the ordered partition of $\langle n\rangle$ such that $\left(A^{g}\right)_{\omega}$ is block lower triangular and $\left(A^{g}\right)_{W_{2} W_{2}}$ is irreducible or a $1 \times 1$ zero block. Then $A_{W_{1} W_{2}}=0$.

Proof. Let $G=A^{g}$. Then

$$
G_{\omega}=\left[\begin{array}{cc}
G_{W_{1} W_{1}} & 0 \\
G_{W_{2} W_{1}} & G_{W_{2} W_{2}}
\end{array}\right],
$$

with $G_{W_{2} W_{2}}$ being irreducible. We will consider two cases: $\rho\left(G_{W_{2} W_{2}}\right)=0$ and $\rho\left(G_{W_{2} W_{2}}\right) \neq 0$.

If $\rho\left(G_{W_{2} W_{2}}\right)=0$, then $G_{W_{2} W_{2}}$ is a $1 \times 1$ block whose entry is zero. Thus there is a nonzero eigenvector $x$ such that $G_{W_{2} W_{2}} x=0 x=0$. Consider the vector

$$
y=\left[\begin{array}{l}
0 \\
x
\end{array}\right],
$$

where 0 is the $(n-1) \times 1$ all zeros vector. Since $\operatorname{index}_{0}(A) \leq 1$, the vector $y$ is also an eigenvector of $A_{\omega}$ corresponding to zero. Then

$$
A_{\omega}\left[\begin{array}{l}
0 \\
x
\end{array}\right]=\left[\begin{array}{c}
A_{W_{1} W_{2}} x \\
A_{W_{2} W_{2}} x
\end{array}\right]=\left[\begin{array}{l}
0 \\
0
\end{array}\right] .
$$

Since $x \neq 0$ and is a scalar, we can conclude $A_{W_{1} W_{2}}=0$. 
If $\rho\left(G_{W_{2} W_{2}}\right) \neq 0$, let $r^{g}=\rho\left(G_{W_{2} W_{2}}\right)$, where by Lemma 3.2, $r \in \sigma(A)$. Since $G_{W_{2} W_{2}} \geq 0$, there exists an $x \gg 0$ such that $G_{W_{2} W_{2}} x=r^{g} x$. This means the vector

$$
y=\left[\begin{array}{l}
0 \\
x
\end{array}\right]
$$

is an eigenvector of $G_{\omega}$ corresponding to the eigenvalue $r^{g}$. Notice the eigenspace of $A$ associated with the eigenvalue $r$ is contained in the eigenspace of $G=A^{g}$ associated with the eigenvalue $r^{g}$, and by the choice of $g$, the two eigenspaces have the same dimension and hence must be equal. Thus $y$ is an eigenvector of $A_{\omega}$ corresponding to $r$ and $y$ is an eigenvector of $A_{\omega}^{g+1}$ corresponding to $r^{g+1}$.

Consider $A_{\omega}^{g+1}=A_{\omega} G_{\omega}$. Then

$$
A_{\omega}^{g+1}\left[\begin{array}{l}
0 \\
x
\end{array}\right]=r^{g+1}\left[\begin{array}{l}
0 \\
x
\end{array}\right]=A_{\omega} G_{\omega}\left[\begin{array}{l}
0 \\
x
\end{array}\right]=\left[\begin{array}{l}
A_{W_{1} W_{2}} G_{W_{2} W_{2}} x \\
A_{W_{2} W_{2}} G_{W_{2} W_{2}} x
\end{array}\right] .
$$

Thus $A_{W_{1} W_{2}} G_{W_{2} W_{2}} x=0$. The submatrix $A_{W_{1} W_{2}} G_{W_{2} W_{2}}$ of $A_{\omega}^{g+1}$ is nonnegative and $x \gg 0$. Thus we conclude $A_{W_{1} W_{2}} G_{W_{2} W_{2}}=0$ and

$$
A_{\omega}^{g+1}=\left[\begin{array}{cc}
A_{W_{1} W_{1}} G_{W_{1} W_{1}}+A_{W_{1} W_{2}} G_{W_{2} W_{1}} & 0 \\
A_{W_{2} W_{1}} G_{W_{1} W_{1}}+A_{W_{2} W_{2}} G_{W_{2} W_{1}} & A_{W_{2} W_{2}} G_{W_{2} W_{2}}
\end{array}\right] .
$$

Let $\left\{x_{1}, \ldots, x_{p}\right\}$ be a union of Jordan bases for the generalized eigenspaces of $G_{W_{2} W_{2}}$. Then $\left\{x_{1}, \ldots, x_{p}\right\}$ is a basis for $\mathbb{C}^{p}$. We want to show $A_{W_{1} W_{2}} x_{j}=0$ for all $j \in\langle p\rangle$. Let $\lambda^{g} \in \sigma\left(G_{W_{2} W_{2}}\right)$. Consider $\lambda^{g}=0$. Because the Jordan blocks corresponding to zero are $1 \times 1$ in $A$, the corresponding vector $x_{j}$ is an eigenvector of $G_{W_{2} W_{2}}$ corresponding to zero. Similar to the preceding case above, we can see that $A_{W_{1} W_{2}} x_{j}=0$.

If $\lambda^{g} \neq 0$, then we consider

$$
A_{\omega}^{g+1}\left[\begin{array}{c}
0 \\
x_{j}
\end{array}\right]=\left[\begin{array}{c}
A_{W_{1} W_{2}} G_{W_{2} W_{2}} x_{j} \\
A_{W_{2} W_{2}} G_{W_{2} W_{2}} x_{j}
\end{array}\right],
$$

which is equal to

(i) $\lambda^{g}\left[\begin{array}{l}A_{W_{1} W_{2}} x_{j} \\ A_{W_{2} W_{2}} x_{j}\end{array}\right]$ if $x_{j}$ is an eigenvector of $G_{W_{2} W_{2}}$; or

(ii) $\left[\begin{array}{l}A_{W_{1} W_{2}}\left(\lambda^{g} x_{j}+x_{j+1}\right) \\ A_{W_{2} W_{2}}\left(\lambda^{g} x_{j}+x_{j+1}\right)\end{array}\right]$ if $x_{j}$ is part of a Jordan chain of $G_{W_{2} W_{2}}$.

Let $x_{j}, x_{j+1}, \ldots, x_{l-1}, x_{l}$ be a Jordan chain in the Jordan basis. We know

$$
\left[\begin{array}{c}
0 \\
x_{l}
\end{array}\right]
$$

is an eigenvector of $A_{\omega}^{g+1}$. By (i) above, we can see that $A_{W_{1} W_{2}} x_{l}=0$. Because $A_{W_{1} W_{2}} x_{l}=0, A_{W_{1} W_{2}}\left(\lambda^{g} x_{l-1}+x_{l}\right)=\lambda^{g} A_{W_{1} W_{2}} x_{l-1}+A_{W_{1} W_{2}} x_{l}=\lambda^{g} A_{W_{1} W_{2}} x_{l-1}=0$. 
Hence $A_{W_{1} W_{2}} x_{l-1}=0$. Using this argument, it can be seen that $A_{W_{1} W_{2}} x_{j}=0$ for all $x_{j}$ in the Jordan chain. Therefore $A_{W_{1} W_{2}} x_{j}=0$ for all $x_{j}$ in a basis for $\mathbb{C}^{p}$. Thus $A_{W_{1} W_{2}}=0$. We conclude that $A_{\omega}=\left[\begin{array}{cc}A_{W_{1} W_{1}} & 0 \\ A_{W_{2} W_{1}} & A_{W_{2} W_{2}}\end{array}\right]$.

Let $A$ be an eventually nonnegative matrix whose Jordan blocks corresponding to zero are at most $1 \times 1$. Applying Lemma 3.3 repeatedly, we find that $A$ has the same Frobenius normal form as $A^{g}$.

TheOREM 3.4. Let $A$ be an eventually nonnegative matrix with $\operatorname{index}_{0}(A) \leq 1$. Let $g$ be a prime number such that $g \notin D_{A}$ and $A^{s} \geq 0$, for all $s \geq g$. Let $\kappa=$ $\left(K_{1}, K_{2}, \ldots, K_{k}\right)$ be the ordered partition of $\langle n\rangle$ such that $A_{\kappa}^{g}$ is in Frobenius normal form. Then $A_{\kappa}$ is in Frobenius normal form. all $j<k$.

Proof. Since $A_{K_{k} K_{k}}^{g}$ is irreducible, by the Lemma 3.3, we see that $A_{K_{j} K_{k}}=0$ for

For $1<l<k$, let $\kappa^{(l)}$ be the ordered partition $\left(K_{1}, \ldots, K_{k-l}\right)$. Consider $A_{\kappa^{(l)}}$. The matrix $A_{\kappa^{(l)}}$ is eventually nonnegative. Because $D_{A_{\kappa^{(l)}}} \subseteq D_{A}, g \notin D_{A_{\kappa^{(l)}}}$. Also $A_{\kappa^{(l)}}^{g}=\left(A_{\kappa^{(l)}}\right)^{g}$. We have that $A_{\kappa^{(l)}}^{g}$ is in Frobenius normal form and $A_{K_{k-l} K_{k-l}}^{g}$ is irreducible. Again by Lemma 3.3, $A_{K_{j} K_{k-l}}=0$ for all $j<k-l$.

In fact, the matrix $A_{\kappa}$ is in Frobenius normal form since, if there were to exist an $A_{K_{j} K_{j}}$ that was reducible, then $\left(A^{g}\right)_{K_{j} K_{j}}$ would be reducible. $\mathrm{Q}$

THEOREM 3.5. Let $A$ be an eventually nonnegative matrix with $\operatorname{index}_{0}(A) \leq 1$. $\underline{L e t} g$ be a prime number such that $g \notin D_{A}$ and $A^{s} \geq 0$, for all $s \geq g$. Then $\overline{R(A)}=$ $\overline{R\left(A^{g}\right)}$ (i.e. they have the same transitive closures).

Proof. Choose $\kappa=\left(K_{1}, K_{2}, \ldots, K_{k}\right)$ such that $\left(A^{g}\right)_{\kappa}$ is in Frobenius normal form. By Theorem 3.4, $A_{\kappa}$ is also in Frobenius normal form. Let $j, l \in\langle k\rangle$ with $j>l$. Since $j>l$, we see that $K_{l}$ does not access $K_{j}$ in $R\left(A^{g}\right)$ or in $R(A)$.

If $K_{j}$ has access to $K_{l}$ in $R\left(A^{g}\right)$ then there must be a path from some vertex $K_{j}$ to some vertex $K_{l}$ in $G(A)$ and hence $K_{j}$ must have access to $K_{l}$ in $R(A)$.

If $K_{j}$ does not have access to $K_{l}$ in $R\left(A^{g}\right)$ then let

$$
\begin{gathered}
Q=\left\{q \mid K_{q} \text { has access to } K_{l} \text { but not } K_{j} \text { in } R\left(A^{g}\right)\right\}, \\
P=\left\{p \mid K_{p} \text { is accessed by } K_{j} \text { in } R\left(A^{g}\right) \text {, but not by } K_{q} \text { for any } q \in Q\right\} .
\end{gathered}
$$

Notice $K_{l} \in Q$ and $K_{j} \in P$. Let $p \in P$ and $q \in Q$. Suppose there is a path from $K_{p}$ to $K_{q}$ in $R\left(A^{g}\right)$. Then there are paths from $K_{j}$ to $K_{p}$, from $K_{p}$ to $K_{q}$, and from $K_{q}$ to $K_{l}$ that together form a path from $K_{j}$ to $K_{l}$, contradicting that $K_{j}$ does not have access to $K_{l}$ in $R\left(A^{g}\right)$. Thus $\left(A^{g}\right)_{P Q}=0$. By our definitions of $P$ and $Q$, we see that $\left(A^{g}\right)_{Q P}=0$. It follows then that there is an ordering of the irreducible classes of $A^{g}$ such that $K_{j}$ appears in the list before $K_{l}$, and for which $A^{g}$ is in Frobenius normal form. By Theorem 3.4, $A$ would also be in Frobenius normal form with respect to this new ordering. Thus if $K_{j}$ does not have access to $K_{l}$ in $R\left(A^{g}\right)$, then $K_{j}$ does not have access to $K_{l}$ in $R(A)$.

Since the access relationships in the two graphs are the same, their transitive closures are equal. 
4. The Combinatorial Structure of Eventually Nonnegative Matrices. We now present a selection of combinatorial spectral properties of nonnegative matrices, which carry over to eventually nonnegative matrices whose index of zero is at most 1. Obviously any nonsingular eventually nonnegative matrix satisfies this property. In Theorem 3.6 [16], they observe there is a decomposition of an eventually nonnegative matrix $A$ into the sum of matrices $B$ and $C$ where $C$ is nilpotent, $B C=C B=0$, and $\operatorname{index}_{0}(B) \leq 1$. The results below hold for the matrix $B$. In the instance where we have an eventually nonnegative matrix $A$ with $1<r=\operatorname{index}_{0}(A)$, we can investigate the spectral properties of $A$ by applying the Corollaries below to the matrix $A^{r}$.

Let $A$ be an eventually nonnegative matrix with $\operatorname{index}_{0}(A) \leq 1$. Let $g$ be a prime number such that $g \notin D_{A}$ and $\lambda \in \sigma(A)$. Observe $J_{l}(\lambda) \in \mathcal{J}(A)$ if and only if $J_{l}\left(\lambda^{g}\right) \in \mathcal{J}\left(A^{g}\right)$ and moreover $E_{\lambda}(A)=E_{\lambda^{g}}\left(A^{g}\right)$. The following results are true for nonnegative matrices, and now extend easily to eventually nonnegative matrices $A$ with $\operatorname{index}_{0}(A) \leq 1$.

We begin by observing that the size of the largest Jordan block associated with $\rho(A)$, in the Jordan form of $A$, is combinatorially determined.

Corollary 4.1. Let $A$ be an eventually nonnegative matrix with $\operatorname{index}_{0}(A) \leq 1$. Let $\rho=\rho(A)$. Then the $\operatorname{index}_{\rho}(A)$ is equal to the maximum level of a vertex in $R(\rho I-A)$.

Proof. Follows from Theorem 3.5 and the nonnegative case as attributed to Rothblum (1975) in [13, Theorem 7.1].

In [6], Hershkowitz and Schneider show the level characteristic of a nonnegative matrix, reordered into nonincreasing order, is majorized by the height characteristic.

COROllary 4.2. Let $A$ be an eventually nonnegative matrix with $\operatorname{index}_{0}(A) \leq 1$. Let $\rho=\rho(A)$ and $\hat{\nu}$ be the ordered sequence formed by listing the elements of $\nu(\rho I-A)$ in nonincreasing order. Then $\hat{\nu} \preceq \eta(\rho I-A)$.

Proof. Follows from Theorem 3.5 and the nonnegative case [6].

Our next result shows there is a nonnegative basis for the generalized eigenspace of $A$, associated with $\rho(A)$, and the positive entries are combinatorially determined.

Corollary 4.3. Let $A$ be an eventually nonnegative matrix with $\operatorname{index}_{0}(A) \leq 1$. Let $\rho=\rho(A)$. Let $\kappa=\left(K_{1}, K_{2}, \ldots, K_{k}\right)$ be such that $A_{\kappa}$ is in Frobenius normal form. Let $p=$ mult $_{\rho}(A)$ and $i_{1}, \ldots, i_{p}$ be chosen such that $\rho\left(A_{K_{i_{j}} K_{i_{j}}}\right)=\rho$. Then there exists a basis $\left\{x^{(1)}, x^{(2)}, \ldots, x^{(p)}\right\}$ of $E_{\rho}(A)$ such that

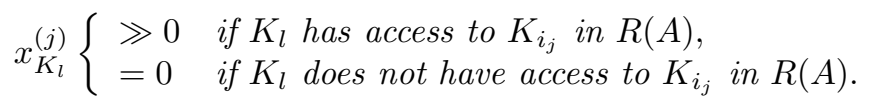

Proof. Follows from Theorem 3.5 and the nonnegative case as attributed to Rothblum (1975) in [13, Theorem 7.1].

Next we characterize the eventually nonnegative matrices $A$ in our class that have a positive right eigenvector associated with $\rho(A)$.

Corollary 4.4. Let $A$ be an eventually nonnegative matrix with $\operatorname{index}_{0}(A) \leq 1$. Let $\rho=\rho(A)$. The following are equivalent:

(i) There exists an $x \gg 0$ such that $(\rho I-A) x=0$. 
(ii) The set of singular vertices of $R(\rho I-A)$ is equal to the set of final vertices of $R(\rho I-A)$.

Proof. Let $g$ be a prime number such that $g \notin D_{A}$ and $A^{s} \geq 0$, for all $s \geq g$.

(i) $\Rightarrow$ (ii): Let $x \gg 0$ and $(\rho I-A) x=0$. Then $\left(\rho^{g} I-A^{g}\right) x=0$. By $[12$, Theorem 2 ], the set of singular vertices of $R\left(\rho^{g} I-A^{g}\right)$ is equal to the set of final vertices of $R\left(\rho^{g} I-A^{g}\right)$. Theorem 3.5 gives that the set singular vertices of $R(\rho I-A)$ is equal to the set of final vertices of $R(\rho I-A)$.

(ii) $\Rightarrow$ (i): Assume the set of singular vertices of $R(\rho I-A)$ is equal to the set of final vertices of $R(\rho I-A)$. By Theorem 3.5, the set of singular vertices of $R\left(\rho^{g} I-A^{g}\right)$ is equal to the set of final vertices of $R\left(\rho^{g} I-A^{g}\right)$. Since $A^{g}$ is nonnegative, there exists $x \gg 0$ such that $\left(\rho^{g} I-A^{g}\right) x=0$. Thus $(\rho I-A) x=0$.

In the next two corollaries we look at nonnegative eigenvectors associated with eigenvalues in addition to the spectral radius.

Corollary 4.5. Let $A$ be an eventually nonnegative matrix with $\operatorname{index}_{0}(A) \leq 1$ and assume $A$ is in Frobenius normal form. Let $\lambda$ be a real number. The following are equivalent:

(i) There exists an eigenvector $x$ such that $A x=\lambda x$ and $x>0$.

(ii) There is a vertex $K_{l}$ of $R(A)$ such that whenever $K_{j}$ has access to $K_{l}$ in $R(A)$, then

$$
\lambda=\rho\left(A_{K_{l} K_{l}}\right)>\rho\left(A_{K_{j} K_{j}}\right) .
$$

Proof. Follows from Theorem 3.5 and the nonnegative case as attributed to Victory (1985) in [13, Theorem 3.7].

Corollary 4.6. Let $A$ be an eventually nonnegative matrix with $\operatorname{index}_{0}(A) \leq 1$ and assume $A$ is in Frobenius normal form. Let $\lambda$ be a real number. If there is a vertex $K_{l}$ of $R(A)$ such that whenever $K_{j}$ has access to $K_{l}$ in $R(A)$, then $\lambda=\rho\left(A_{K_{l} K_{l}}\right)>$ $\rho\left(A_{K_{j} K_{j}}\right)$, there is a (up to scalar multiples) unique vector $x$ that satisfies $A x=\lambda x$ and

$$
x_{K_{j}} \begin{cases}\gg 0 & \text { if } K_{j} \text { has access to } K_{l} \text { in } R(A) . \\ =0 & \text { if } K_{j} \text { does not have access to } K_{l} \text { in } R(A) .\end{cases}
$$

Proof. Follows from Theorem 3.5 and the nonnegative case as attributed to Victory (1985) in [13, Theorem 3.7].

We conclude our list of combinatorial properties of nonnegative matrices, which carry over to our class of eventually nonnegative matrices, with one final corollary. The combinatorial spectral properties of nonnegative matrices is a very rich area. We recognize that our list is by no means exhaustive and encourage readers to check if their favourite properties also hold.

Corollary 4.7. Let $A$ be an eventually nonnegative matrix with $\operatorname{index}_{0}(A) \leq 1$. Let $\rho=\rho(A)$. Then the following are equivalent:

(i) For any vector $z$, the vector $(\rho I-A) z \geq 0$ implies that the vector $(\rho I-A) z=0$.

(ii) The set of initial vertices of $R(\rho I-A)$ is equal to the set of singular vertices of $R(\rho I-A)$. 
Proof. not (ii) $\Rightarrow$ not (i):

Case I: There exists an initial class $K$ of $R(A)$ such that $\lambda=\rho\left(A_{K K}\right)<\rho(A)$. Choose $x \gg 0$ such that $A_{K K} x=\lambda x$. Let $W=\langle n\rangle / K$ and $\omega=(W, K)$. Then

$$
\left(\rho I-A_{\omega}\right)\left[\begin{array}{l}
0 \\
x
\end{array}\right]=\rho\left[\begin{array}{l}
0 \\
x
\end{array}\right]-\lambda\left[\begin{array}{l}
0 \\
x
\end{array}\right]=(\rho-\lambda)\left[\begin{array}{l}
0 \\
x
\end{array}\right]>0 .
$$

Set

$$
z_{\omega}=\left[\begin{array}{l}
0 \\
x
\end{array}\right]
$$

Then $\left(\rho I-A_{\omega}\right) z_{\omega}>0$ follows.

Case II: Every initial class has spectral radius $\rho$, but there exists a noninitial class with spectral radius $\rho$. By Corollary 4.1 and Theorem 3.5, $h=\operatorname{index}_{\rho}(A) \geq 2$ and $h=\operatorname{index}_{\rho^{g}}\left(A^{g}\right) \geq 2$. By [13, Corollary 7.6], there exists a Jordan chain for $A^{g}$ consisting of $h$ nonnegative vectors. Let $x$ generate the chain. Then $(\rho I-A)^{h-1} x$ is a positive scalar multiple of $\left(\rho^{g} I-A^{g}\right)^{h-1} x$ (look at the change of basis matrix that takes $\left(J_{l}(\rho)\right)^{g}$ to $\left.J_{l}\left(\rho^{g}\right)\right)$. So $z=(\rho I-A)^{h-2} x$ has the property that $(\rho I-A) z>0$.

(ii) $\Rightarrow$ (i): The graph $R\left(\rho I-A^{T}\right)$ satisfies Corollary 4.4 , so there exists a $y \gg 0$ such that $y^{T}(\rho I-A)=0$. If $(\rho I-A) z>0$, then $y^{T}[(\rho I-A) z]>0$. But $\left[y^{T}(\rho I-A)\right] z=0$, a contradiction. $\square$

We conclude our paper with a few more examples.

EXAMPLE 4.8. We now look at two eventually nonnegative matrices that appear in [15]. The matrices

$$
A^{(1)}=\left[\begin{array}{cc|cc|cc}
2 & 2 & 0 & 0 & 0 & 0 \\
2 & 2 & 0 & 0 & 0 & 0 \\
\hline 1 & 1 & 1 & 1 & 0 & 0 \\
1 & 1 & 1 & 1 & 0 & 0 \\
\hline 0 & 0 & 1 & -1 & 2 & 2 \\
0 & 0 & -1 & 1 & 2 & 2
\end{array}\right] \text { and } A^{(2)}=\left[\begin{array}{cc|cccc}
2 & 2 & 0 & 0 & 0 & 0 \\
2 & 2 & 0 & 0 & 0 & 0 \\
\hline 1 & 1 & 2 & 0 & -1 & 1 \\
1 & 1 & 0 & 2 & 1 & -1 \\
0 & 0 & 1 & -1 & 1 & 3 \\
0 & 0 & -1 & 1 & 3 & 1
\end{array}\right]
$$

have index ${ }_{0}\left(A^{(j)}\right)=2$. Both matrices have vertices in $R\left(4 I-A^{(j)}\right)$ with level 2 even though index $4\left(A^{(j)}\right)=1$. If, however, we raise each matrix to the power of the $\operatorname{index}_{0}\left(A^{(j)}\right)$ we see that

$$
\left(A^{(1)}\right)^{2}=\left(A^{(2)}\right)^{2}=\left[\begin{array}{cc|cc|cc}
8 & 8 & 0 & 0 & 0 & 0 \\
8 & 8 & 0 & 0 & 0 & 0 \\
\hline 6 & 6 & 2 & 2 & 0 & 0 \\
6 & 6 & 2 & 2 & 0 & 0 \\
\hline 0 & 0 & 0 & 0 & 8 & 8 \\
0 & 0 & 0 & 0 & 8 & 8
\end{array}\right]
$$

which obviously exhibits the standard combinatorial structure of a nonnegative matrix. 
Example 4.9. Consider the matrix $A$ below that is 1-cyclic and eventually nonnegative. The matrix $A^{6}$ is the direct sum of six positive matrices. We will define $A$ to be the sum of two matrices $B$ and $C$ such that $\operatorname{index}_{0}(B) \leq 1, C$ is nilpotent, and $B C=C B=0$.

Let $T$ be the $2 \times 2$ matrix of all twos. Let

$$
R=\left[\begin{array}{rr}
1 & -1 \\
-1 & 1
\end{array}\right]
$$

We define $B$ and $C$ as follows:

$$
B=\left[\begin{array}{rrrrrr}
0 & T & 0 & 0 & 0 & 0 \\
T & 0 & 0 & 0 & 0 & 0 \\
0 & 0 & 0 & T & 0 & 0 \\
0 & 0 & 0 & 0 & T & 0 \\
0 & 0 & T & 0 & 0 & 0 \\
0 & 0 & 0 & 0 & 0 & T
\end{array}\right], \quad C=\left[\begin{array}{rrrrrr}
0 & 0 & R & R & R & -R \\
0 & 0 & -R & -R & R & -R \\
0 & 0 & 0 & 0 & 0 & 0 \\
0 & 0 & 0 & 0 & 0 & 0 \\
R & -R & 0 & 0 & 0 & 0 \\
R & -R & 0 & 0 & 0 & 0
\end{array}\right]
$$

with $A=B+C$.

The matrix $A$ is an irreducible eventually nonnegative matrix. Once $A$ is raised to power $3=\operatorname{index}_{0}(A), A^{3}$ can be partitioned into the direct sum of nonnegative matrices. In particular, any prime number $g>3$ is not an element of $D_{A}=\{ \pm 2, \pm 3, \pm 6\}$ and so the Frobenius normal form of $A^{g}$ is the same as the Frobenius normal form of $B$ in the decomposition of $A$.

For example, seven is not an element in $D_{A}$. Consider

$$
A^{7}=B^{7}=\left[\begin{array}{cccc|cccccc|cc}
0 & 0 & 2^{13} & 2^{13} & 0 & 0 & 0 & 0 & 0 & 0 & 0 & 0 \\
0 & 0 & 2^{13} & 2^{13} & 0 & 0 & 0 & 0 & 0 & 0 & 0 & 0 \\
2^{13} & 2^{13} & 0 & 0 & 0 & 0 & 0 & 0 & 0 & 0 & 0 & 0 \\
2^{13} & 2^{13} & 0 & 0 & 0 & 0 & 0 & 0 & 0 & 0 & 0 & 0 \\
\hline 0 & 0 & 0 & 0 & 0 & 0 & 2^{13} & 2^{13} & 0 & 0 & 0 & 0 \\
0 & 0 & 0 & 0 & 0 & 0 & 2^{13} & 2^{13} & 0 & 0 & 0 & 0 \\
0 & 0 & 0 & 0 & 0 & 0 & 0 & 0 & 2^{13} & 2^{13} & 0 & 0 \\
0 & 0 & 0 & 0 & 0 & 0 & 0 & 0 & 2^{13} & 2^{13} & 0 & 0 \\
0 & 0 & 0 & 0 & 2^{13} & 2^{13} & 0 & 0 & 0 & 0 & 0 & 0 \\
0 & 0 & 0 & 0 & 2^{13} & 2^{13} & 0 & 0 & 0 & 0 & 0 & 0 \\
\hline 0 & 0 & 0 & 0 & 0 & 0 & 0 & 0 & 0 & 0 & 2^{13} & 2^{13} \\
0 & 0 & 0 & 0 & 0 & 0 & 0 & 0 & 0 & 0 & 2^{13} & 2^{13}
\end{array}\right] .
$$

This matrix has the same reducible structure as the matrix $B$ in the decomposition of $A$.

The focus of this paper has been combinatorial properties of reducible nonnegative matrices, which carry over to eventually nonnegative matrices, where the index of zero is at most 1 . In our last example, we show how to construct nonsingular irreducible eventually positive matrices $A$ so the smallest $g$ for which $A^{g} \gg 0$, depends on quantitative rather than qualitative properties of $A$. Thus results on exponents 
of primitive matrices (see [1]) will not carry over to eventually nonnegative matrices with index 01 .

Example 4.10. Given any odd integer $N>0$, we construct an $n \times n$ matrix $A$ such that $A^{N}$ is not positive, but $A^{s} \gg 0$ for all $s>N$. We use a method due to Soules [14], and generalized in [2], to illustrate how to construct a matrix $A$ with prescribed eigenvalues. Let

$$
\mathcal{T}_{n}=\left\{\left(\lambda_{1}, \lambda_{2}, \ldots, \lambda_{n}\right) \mid 1=\lambda_{1} \geq \lambda_{2}, \ldots, \lambda_{n} \geq-1, \quad \lambda_{1}+\lambda_{2}+\ldots+\lambda_{n} \geq 0\right\}
$$

be a subset of $\mathbb{R}^{n}$. Let $D$ be the diagonal matrix

$$
D=\left[\begin{array}{ccccc}
\lambda_{1} & 0 & \ldots & \ldots & 0 \\
0 & \lambda_{2} & 0 & \ldots & 0 \\
\vdots & \ddots & \ddots & \vdots & \vdots \\
0 & \ldots & 0 & \lambda_{n-1} & 0 \\
0 & \ldots & \ldots & 0 & \lambda_{n}
\end{array}\right]
$$

Then from the work in [9], we know that for each Soules matrix $R$, there is a closed convex polytope $\mathcal{S}(R) \subseteq \mathcal{T}_{n}$, for which $A=R D R^{T} \geq 0$ for $\left(\lambda_{1}, \lambda_{2}, \ldots, \lambda_{n}\right) \in \mathcal{T}_{n}$ if and only if $\left(\lambda_{1}, \lambda_{2}, \ldots, \lambda_{n}\right) \in \mathcal{S}(R)$. The point $(1,0, \ldots, 0) \in \mathcal{S}(R)$, so if $\left|\lambda_{j}\right|<1$ for all $1<j \leq n$, then for any sufficiently large positive integer $s$, the point $\left(1, \lambda_{2}^{s}, \ldots, \lambda_{n}^{s}\right) \in$ $\mathcal{S}(R)$, even though $\left(\lambda_{1}, \lambda_{2}, \ldots, \lambda_{n}\right) \notin \mathcal{S}(R)$. In this manner we can easily construct symmetric eventually nonnegative matrices that become nonnegative matrices at a prescribed exponent. We now pick a particular Soules matrix $R$, and choose our eigenvalues for $A$ such that $A^{N}$ is nonnegative, and this is the first odd exponent for which this happens. For this particular example, we will go on to show that $A^{s} \gg 0$, for $s \geq N+1$, but the diagonal elements of $A^{N}$ are zero. Consider the Soules matrix

$$
R=\left[\begin{array}{cccccc}
\frac{1}{\sqrt{n}} & \frac{1}{\sqrt{n(n-1)}} & \frac{1}{\sqrt{(n-1)(n-2)}} & \ldots & \ldots & \frac{1}{\sqrt{2}} \\
\frac{1}{\sqrt{n}} & \frac{1}{\sqrt{n(n-1)}} & \frac{1}{\sqrt{(n-1)(n-2)}} & \cdots & \ldots & -\frac{1}{\sqrt{2}} \\
\vdots & \vdots & \vdots & & & 0 \\
\frac{1}{\sqrt{n}} & \frac{1}{\sqrt{n(n-1)}} & \frac{1}{\sqrt{(n-1)(n-2)}} & & & \vdots \\
\frac{1}{\sqrt{n}} & \frac{1}{\sqrt{n(n-1)}} & \frac{2-n}{\sqrt{(n-1)(n-2)}} & & & \vdots \\
\frac{1}{\sqrt{n}} & \frac{1-n}{\sqrt{n(n-1)}} & 0 & \ldots & \ldots & 0
\end{array}\right] .
$$


Let $\delta$ be a positive parameter with $0<\delta \leq 1$, and let

$$
D=\left[\begin{array}{ccccc}
1 & 0 & \ldots & \ldots & 0 \\
0 & -\delta & 0 & \ldots & 0 \\
\vdots & \ddots & \ddots & \vdots & \vdots \\
0 & \ldots & 0 & -\delta & 0 \\
0 & \ldots & \ldots & 0 & -\delta
\end{array}\right]
$$

Let $A=R D R^{T}$. Then $A^{s}=R D^{s} R^{T}$

$$
=\left[\begin{array}{ccccc}
\frac{1+(n-1)(-\delta)^{s}}{n} & \frac{1-(-\delta)^{s}}{n} & \ldots & \ldots & \frac{1-(-\delta)^{s}}{n} \\
\frac{1-(-\delta)^{s}}{n} & \frac{1+(n-1)(-\delta)^{s}}{n} & \frac{1-(-\delta)^{s}}{n} & \ldots & \frac{1-(-\delta)^{s}}{n} \\
\vdots & \ddots & \ddots & \ddots & \vdots \\
\frac{1-(-\delta)^{s}}{n} & \ldots & \frac{1-(-\delta)^{s}}{n} & \frac{1+(n-1)(-\delta)^{s}}{n} & \frac{1-(-\delta)^{s}}{n} \\
\frac{1-(-\delta)^{s}}{n} & \ldots & \ldots & \frac{1-(-\delta)^{s}}{n} & \frac{1+(n-1)(-\delta)^{s}}{n}
\end{array}\right] .
$$

Setting $\delta=\left(\frac{1}{n-1}\right)^{\frac{1}{N}}$, we see that the diagonal elements of $A^{N}$ are zero. Notice $A^{N} \geq 0$ and $A^{s} \gg 0$, for $s \geq N+1$. The eigenvalues of $A^{s}$ are $\left\{1,(-\delta)^{s}, \ldots,(-\delta)^{s}\right\}$.

\section{REFERENCES}

[1] A. Berman and R.J. Plemmons. Nonnegative Matrices in the Mathematical Sciences. Academic Press, New York, 1979.

[2] L. Elsner, R. Nabben, and M. Neumann. Orthogonal bases that lead to symmetric, nonnegative matrices. Linear Algebra and Its Applications, 271:323-343, 1998.

[3] S. Friedland. On an inverse problem for nonnegative and eventually nonnegative matrices. Israel Journal of Mathematics, 29:43-60, 1978.

[4] D. Handelman. Positive matrices and dimension groups affiliated to $C^{*}$-algebras and topological Markov chains. Journal of Operator Theory, 6:55-74, 1981.

[5] D. Hershkowitz. The combinatorial structure of generalized eigenspaces - from nonnegative matrices to general matrices. Linear Algebra Its Applications, 302/303:173-191, 1999.

[6] D. Hershkowitz and H. Schneider. Combinatorial bases, derived Jordan sets and the equality of the height and level characteristics of an M-matrix. Linear Algebra Its Applications, 29:21-42, 1991.

[7] D. Hershkowitz and H. Schneider. On the existence of matrices with prescribed height and level characteristics. Israel Journal of Mathematics, 75:105-117, 1991.

[8] C. Knudsen and J.J. McDonald. A note on the convexity of the realizable set of eigenvalues for nonnegative symmetric matrices. Electronic Journal of Linear Algebra, 8:110-114, 2001.

[9] J.J. McDonald and M. Neumann. The Soules approach to the inverse eigenvalue problem for nonnegative symmetric matrices of order $n \leq 5$. Contemporary Mathematics, 259:387-390, 2000 .

[10] D. Richman and H. Schneider. On the singular graph and the Weyr characteristic of an Mmatrix. Aequationes Mathematicae, 17:208-234, 1978.

[11] U.G. Rothblum. Algebraic eigenspaces of nonnegative matrices. Linear Algebra Its Applications, 12:281-292, 1975. 
[12] U.G. Rothblum. A rank characterization of the number of final classes of a nonnegative matrix. Linear Algebra and Its Applications, 23:65-68, 1979.

13] H. Schneider. The influence of the marked reduced graph of a nonnegative matrix on the Jordan form and on related properties: A survey. Linear Algebra and Its Applications, 84:161-189, 1986.

[14] G.W. Soules. Constructing nonnegative symmetric matrices. Linear and Multilinear Algebra, 13:241-251, 1983.

[15] B.G. Zaslavsky and J.J. McDonald. A Characterization of Jordan canonical forms which are similar to eventually nonnegative matrices. Submitted to Linear Algebra and Its Applications.

[16] B.G. Zaslavsky and B.-S. Tam. On the Jordan form of an irreducible matrix with eventually nonnegative powers. Linear Algebra and Its Applications, 302/303:303-330, 1999. 